\title{
RELATIVISTIC SPIN NETWORKS AND QUANTUM GRAVITY
}

\author{
JOHN W. BARRETT \\ LOUIS CRANE
}

12 September 1997; revised 21 January 1998

\begin{abstract}
Relativistic spin networks are defined by considering the spin covering of the group $\mathrm{SO}(4), \mathrm{SU}(2) \times \mathrm{SU}(2)$. Relativistic quantum spins are related to the geometry of the 2-dimensional faces of a 4 -simplex. This extends the idea of Ponzano and Regge that $\mathrm{SU}(2)$ spins are related to the geometry of the edges of a 3-simplex. This leads us to suggest that there may be a 4-dimensional state sum model for quantum gravity based on relativistic spin networks which parallels the construction of 3-dimensional quantum gravity from ordinary spin networks.
\end{abstract}

PACS 04.60.Nc. 


\section{INTRODUCTION}

In [1] and [2], it was proposed that the quantum theory of gravity could take the form of a very special type of discrete model on a triangulated 4-manifold, which has the property that the propagation of physical states does not depend on the choice of triangulation. Such a model is called a topological state sum.

Topological state sums are closely related to topological quantum field theories. Arguments that the quantum theory of gravity should be closely related to a topological quantum field theory are given in [3].

In [4] a specific family of four dimensional topological state sums were constructed. It was suggested in [1] and [2] that the topological state sum of [4] might be a representation of a topological state for the quantum theory of gravity, and that the physical hypothesis that the universe is in that state could be studied.

Ooguri [5], and more recently, Baez [6] have argued that the topological state sum of [4] (reformulated in [7]), which is a precise realisation of [5], is a construction of the quantum theory of the $B F$ lagrangian.

In fact, the Plebanski Lagrangian $[8,9]$ for general relativity has the form of $B F$ theory [10] with gauge group $\operatorname{SL}(2, C)$, with an added term which is a constraint. Smolin [11] has argued that for general relativity with a cosmological constant the constraint vanishes for the self-dual sector. This suggests $[1,2]$ that the self-dual sector can be identified with the topological state sum of [4].

The purpose of this paper is to outline a new state sum model which is a sort of constrained doubling of the topological state sum of [4]. This constraint is a quantum version of the Plebanski constraint.

In three dimensions, quantum gravity is constructed by the Ponzano-Regge state sum model in which representations of $\mathrm{SU}(2)$ label edges [12]. This corresponds to a path integral in which the metric is the variable. However this model can also be understood as a path integral in the first-order formalism using a frame field and connection form. [13]

It is worth pursuing the link with the classical frame field to get a heuristic understanding of the discrete models. Integrating the frame field $e$, an $\mathbb{R}^{3}$-valued 1 -form, along an edge gives a vector in $\mathbb{R}^{3}$. This can also be regarded as an element of the dual of the Lie algebra so(3).

The idea is that to pass from 3 dimensions to 4 dimensions one has to replace $\mathrm{SO}(3)$ with $\mathrm{SO}(4)$, or its spin covering, $\mathrm{SU}(2) \times \mathrm{SU}(2)$.

The topological home for this spin is now on triangles. This accords with the assignment of spins to triangles in [4], which can be regarded as a special case of $\mathrm{SU}(2) \times \mathrm{SU}(2)$, where one of the copies of $\mathrm{SU}(2)$ acts trivially, thus rendering all variables self-dual. We are also making contact with the loop variables picture of Rovelli and Smolin [14], in which the fundamental operators live on surfaces. The relation of the loop variables to state sum models, and the difference between $B F$ theory and gravity in this context, was investigated in [15] and [16].

In a $B F$ theory, the $B$ field is an so $(4)^{*}$-valued 2 -form, which can be integrated on a triangle to give an element of $\mathrm{so}(4)^{*}$, the dual of the Lie algebra so(4). However, in Einstein gravity it is important that so(4)* is identified with $\mathbb{R}^{4} \wedge \mathbb{R}^{4}$, as $B$ is constrained to be of the form $B=* e \wedge e$, where $e$ is a vector valued one-form. In this 
formula, $e \wedge e$ is an $\mathbb{R}^{4} \wedge \mathbb{R}^{4}$-valued 2-form, and $*$ is the Hodge $*: \mathbb{R}^{4} \wedge \mathbb{R}^{4} \rightarrow \mathbb{R}^{4} \wedge \mathbb{R}^{4}$.

Assuming the frame field gives a linear embedding of the triangle in $\mathbb{R}^{4}$, the integral over this triangle,

$$
\int e \wedge e
$$

is a simple bivector, i.e., of the form $f \wedge g \in \mathbb{R}^{4} \wedge \mathbb{R}^{4}$. Thus the essential constraint which marks Einstein gravity from $B F$ theory is the simplicity of bivectors.

This remark is the key to our construction of the discrete model, even though we do not have a classical frame field in the construction. The discrete models and the path integral formalism with classical frame fields do have close parallels, however.

A discretisation of the classical Einstein gravity using bivectors on triangles which are constrained to be simple was previously studied in $[17,18]$. Some related discrete classical models with self-dual bivectors, rather than simple bivectors, were studied in $[18,19]$.

The new state sum model gives a quantum mechanical version of these constraints. The translation is to replace bivectors with bivector operators. The simplicity condition is then translated into a condition in the representation theory for these operators.

At present we have an axiomatic description of the space of vertices but not an explicit construction. If the only allowable vertex is the one we explicitly construct, then the state sum does not seem to give a topological invariant of manifolds. Thus the relationship of our new state sum to topology is still unclear.

However, it is already very close to a topological theory, in that the deviation from the topological theory [4] is only in the imposed constraints. This should make investigation of its 'ultraviolet' behavior much simpler than for a continuum theory. Our hope is that the 'doubling' of the topological state sum of [4] should allow us to pass from the self-dual sector to the full quantum theory of gravity.

The new state sum has a very natural relationship with Regge calculus in a new formulation, in that the classical version of the theory has the metric as its variable in the same way as Regge calculus. We believe, though do not have a complete argument, that it will contain 'local degrees of freedom' as does general relativity and that propagating plane waves will occur in a linearization of the theory as in $[20,21]$. However we do not yet have an analogue of the Ponzano-Regge asymptotic formula which would give a direct link with the Einstein action.

The heart of our construction is a reformulation of the geometry of simplexes in Euclidean (or Minkowski) 4-space in terms of variables on their 2-subsimplexes. This paper, accordingly, begins with a theorem in classical 4-dimensional Euclidean geometry. The theorem allows us to describe 4-simplexes in terms of oriented area variables on 2-simplexes, giving a more precise version of the idea in [17]. The constraints on the variables have a very elegant form, which can be carried over to the quantum theory.

Having discovered this form for the quantum constraints, it then becomes relatively straightforward to produce the new state sum. 


\section{BiveCtor GEOMETRY OF A 4-SIMPLEX}

An abstract 4-simplex is determined by an ordered set of 5 points, which we denote (01234). The faces of the abstract 4-simplex are just the subsets of this set.

A geometric 4-simplex is the convex hull of 5 points in $\mathbb{R}^{4}$. This embedding is required to be non-degenerate, i.e., the 5 points do not lie in any hyperplane. To each face now corresponds an affine subspace in $\mathbb{R}^{4}$, and also a linear subspace through the origin in $\mathbb{R}^{4}$ which is parallel to it. In particular, each triangle (2simplex) determines a plane through the origin.

An embedding of an abstract 4-simplex in $\mathbb{R}^{4}$ is simply the set of position coordinates $x_{0}, x_{1}, x_{2}, x_{3}, x_{4} \in \mathbb{R}^{4}$ which determine the vertices of a geometric 4 -simplex. The ordering of the vertices also determines an orientation for the simplex.

An oriented triangle in $\mathbb{R}^{4}$ determines a bivector. The position coordinates $x_{0}, x_{1}, x_{2} \in \mathbb{R}^{4}$ determine the displacement vectors for the edges $e_{01}=x_{0}-x_{1}$, $e_{02}=x_{0}-x_{2}$ and hence the bivector $b=\frac{1}{2} e_{01} \wedge e_{02} \in \mathbb{R}^{4} \wedge \mathbb{R}^{4}$. This bivector is invariant under an even permutation of the vertices but changes sign for an odd permutation.

In this way, a geometric 4-simplex determines a set of 10 bivectors, one for each triangle. We show that the bivectors characterise the geometric 4-simplex uniquely, and give the conditions that an assignment of 10 bivectors to the triangles of an abstract 4-simplex determines an embedding.

The properties of the bivectors for a 4-simplex are

(1) The bivector changes sign if the orientation of the triangle is changed.

(2) Each bivector is simple, i.e. of the form $b=f \wedge g$.

(3) If two triangles share a common edge, then the sum of the two bivectors is also simple.

(4) The sum of the 4 bivectors corresponding to the faces of a tetrahedron is zero. This sum is calculated using the orientation of the triangles given by the boundary of the tetrahedron.

(5) The assignment of bivectors is non-degenerate. This means that for six triangles sharing a common vertex, the six bivectors are linearly independent.

(6) Using the Euclidean metric, the bivectors can be considered as linear operators. Then for 3 triangles meeting at a vertex of a tetrahedron, $\operatorname{Tr} b_{1}\left[b_{2}, b_{3}\right]>$ 0 . The sign in this formula is determined by a convention relating a choice for the orientation for the boundary of the tetrahedron to the order in which the three bivectors appear in this formula.

Conditions (2), (3) and (4) were introduced in [17]. We are indebted to John Baez [22] who pointed out the necessity of (6) and the inversion ambiguity in the theorem which were missing in our first version of this paper.

Theorem. Each geometric 4-simplex determines a set of bivectors satisfying these conditions, and each set of bivectors satisfying these conditions determines a geometric 4-simplex unique up to parallel translation and inversion through the origin.

Proof. A simple bivector determines a plane (of dimension 2) through the origin in $\mathbb{R}^{4}$. Two simple bivectors $b_{1}, b_{2}$ determine two planes lying in a hyperplane (of 
dimension 3) if and only if $b_{1}+b_{2}$ is simple. If the planes lie in a hyperplane, this is clear because all bivectors are simple in three dimensions. Conversely, the simplicity condition can be understood as $b \wedge b=0$. Then

$$
0=\left(b_{1}+b_{2}\right) \wedge\left(b_{1}+b_{2}\right)=2 b_{1} \wedge b_{2} .
$$

If $b_{1}=x \wedge y, b_{2}=z \wedge t$, then $0=x \wedge y \wedge z \wedge t$ implies a linear relation between $x, y, z, t$. This calculation also shows that (3) is insensitive to the orientation of the triangles.

For the bivectors for a geometric 4-simplex (4) is satisfied by Stokes' theorem. Condition (5) is true because the edges meeting at a vertex can be taken as axes for $\mathbb{R}^{4}$, and then the bivectors are clearly linearly independent. Condition (6) follows from the calculation $\operatorname{Tr} b_{1}\left[b_{2}, b_{3}\right]=\frac{9}{8} V^{2}$, where $V$ is the volume of the tetrahedron [23]. This completes the proof that the conditions are satisfied by a geometric 4-simplex.

Now suppose a set of bivectors $b_{i}$ satisfies (1)-(6). The simplicity conditions and the non-degeneracy imply that the planes corresponding to the four faces of a tetrahedron either all lie in a common hyperplane or share a common direction. However if the bivectors share a common direction, then $\operatorname{Tr} b_{1}\left[b_{2}, b_{3}\right]=0$, contradicting (6). Therefore the four planes for a tetrahedron lie in a hyperplane. Furthermore, each plane is determined as the intersection of two of these hyperplanes, as each triangle is contained in two tetrahedra. The non-degeneracy condition implies that the five hyperplanes intersect generically, that is, any four of them intersect at a point.

To construct the geometric 4-simplex, shift one of the five hyperplanes away from the origin by parallel translation. The hyperplanes now bound a geometric 4-simplex, with 2-dimensional faces parallel to the planes corresponding to the original bivectors $b_{i}, i=1, \ldots 10$. This simplex has 10 bivectors $b_{i}^{\prime}=\lambda_{i} b_{i}$ for some 10 scalars $\lambda_{i} \neq 0 \in \mathbb{C}$. Condition (4) is the only linear relation on the bivectors for the faces of a tetrahedron. This implies that the $\lambda_{i}$ are all equal for the faces of a tetrahedron, and hence are all equal for all ten triangles.

The geometric 4-simplex can be scaled by moving the hyperplane. If its distance from the origin is scaled by a parameter $\mu$, then the bivectors all scale as $\mu^{2}$. Therefore the $\lambda_{i}$ can be scaled so that they are all equal to 1 or all equal to -1 . Then the area bivectors $b_{i}^{\prime}$ of the reconstructed 4 -simplex are either exactly equal to the original $b_{i}$, or all equal to $-b_{i}$. However the $b_{i}^{\prime}$ also satisfy $(6)$ as they are the bivectors of a geometric 4-simplex. Hence the inequality in (6) forces the $b_{i}^{\prime}$ to be exactly equal to the $b_{i}$ and not to their negatives.

The scaling parameter $\mu$ is only determined up to a sign, and so the geometric 4 -simplex is determined up to inversion about the origin. This operation does not affect the area bivectors.

Metric geometry. Now introduce the standard Euclidean metric on $\mathbb{R}^{4}$ (One could also use the standard Minkowski metric). If the bivectors are replaced by their duals, $* b$, then conditions $(1)-(5)$ are still satisfied but $\operatorname{Tr} b_{1}\left[b_{2}, b_{3}\right]=0$. This duality interchanges a set of planes lying in a hyperplane with a set of planes sharing a common direction. 
The bivectors can be identified with elements of the Lie algebra so(4). (Also, it may be profitable to think of them as the elements of the dual of this Lie algebra.) The splitting so(4) $\simeq \mathrm{su}(2) \oplus \mathrm{su}(2)$ is then the same as the splitting of the space of bivectors into self-dual and anti-self-dual parts, $\mathbb{R}^{4} \wedge \mathbb{R}^{4}=\Lambda_{+}^{2} \oplus \Lambda_{-}^{2}$. The condition that a bivector $b$ is simple is

$$
0=<b, * b>=<b^{+}, b^{+}>-<b^{-}, b^{-}>,
$$

so that the norm of the self-dual and anti-self-dual parts is equal. The area $A$ of the triangle is determined by

$$
A^{2}=<b, b>=<b^{+}, b^{+}>+<b^{-}, b^{-}>\text {. }
$$

A geometric simplex has a metric geometry, which is determined by the lengths of its 10 edges. Thus two geometric 4-simplexes have the same edge lengths if and only if they are related by an isometry of $\mathbb{R}^{4}$. Therefore the bivector data determines a set of edge lengths. Two sets of bivector data determine the same edge lengths if and only if the bivectors are all transformed simultaneously by an orthogonal rotation.

By contrast, specifying the 10 areas does not specify the metric geometry uniquely [24], although it does for almost all configurations. However giving the areas of the four faces of a tetrahedron does not specify the geometry of the tetrahedron uniquely, so that two 4-simplexes cannot be glued together by specifying the areas of the 16 triangles in their union [25]. For this reason, we decided that the theory should be based on bivectors rather than areas.

\section{RELATIVISTIC SPIN NETWORKS}

In the theory of spin networks, using the Lie group $\mathrm{SU}(2)$, each representation determines a map from vectors in $\mathbb{R}^{3}$ to operators in the representation space, namely the representation of the Lie algebra. In quantum physics, these are called vector operators. The intuition is that in the classical limit, when the representation is 'large', these behave as ordinary vectors in 3-dimensional space, with probability near to 1.

Given three irreducible representations on spaces $V_{a}, V_{b}, V_{c}$, the spin network trivalent vertex is a uniquely determined element of

$$
V_{a} \otimes V_{b} \otimes V_{c}
$$

In a three-dimensional theory, this is the vertex for a triangle. This element is invariant under the group action. The corresponding vector operators satisfy

$$
J_{a}+J_{b}+J_{c}=0
$$

when applied to this state, as a consequence. This is the relation for the edge vectors of a triangle, or, equivalently, the bivectors in $\mathbb{R}^{3}$ which are dual to the edge vectors. Note that for other groups, the space of vertices may form a vector 
space of dimension greater than one. In these cases, it is necessary to pick a basis for these spaces and sum over the basis in a state sum model.

To define a theory of relativistic spin networks, we label the edges of a graph with irreducible representations of $\mathrm{SU}(2) \times \mathrm{SU}(2)$. These are pairs $(j, k)$ of representations of $\mathrm{SU}(2)$. Then the representation of the Lie algebra gives bivector operators, by analogy with the preceding case. The condition that these are simple bivectors can be transcribed into the quantum theory by requiring that $j=k$. This is the correct condition because classically it is requiring the self-dual and anti-self-dual parts to have the same norm.

We also have in mind using the $q$-deformation of spin networks [26]. This is algebraically very similiar to the undeformed case, with the advantage that the computations can be made finite by the choice of a root of unity. A disadvantage is the loss of the simple geometrical picture of bivectors. Also, the representations are braided. We take the braiding for the two copies of $\mathrm{SU}(2)$ to be opposite, i.e., related by an orientation reversing map.

Now in the relativistic case, the theory is for four-dimensional manifolds and the relativistic spin network vertices reside in a tetrahedron. There will be one bivector operator for each face of a tetrahedron, and so this will be a four-valent vertex. The idea for relating spins on faces to the geometry of a tetrahedron is from Barbieri [23], who considered a single $\mathrm{SU}(2)$ spin.

The labelling of a 4-simplex is as follows. The triangles are labelled with representations of $\mathrm{SU}(2) \times \mathrm{SU}(2)$, and each tetrahedron is labelled with a tensor in the product of the four spaces on its faces.

(1) Changing the orientation of a triangle changes the representation to its dual.

(2) The representations on the triangles are of the form $(j, j)$. These are called simple representations.

(3) For any pair of faces of a tetrahedron, the pair of representations can be decomposed into its Clebsch-Gordan series for $\mathrm{SU}(2) \times \mathrm{SU}(2)$. Under this isomorphism, the tensor for the tetrahedron decomposes into summands which are non-zero only for the simple representations of $\mathrm{SU}(2) \times \mathrm{SU}(2)$.

(4) The tensor for the tetrahedron is an invariant tensor.

These conditions are the quantum analogues of the properties (1) - (4) of bivectors on a 4-simplex given earlier. For the first condition, note that in the case of a classical Lie algebra, the action of the Lie algebra in the dual representation is minus the adjoint of the original action. We have not analysed the inequalities (5) and (6), but note that a simple operator analogue of (6) seems feasible. Operator versions of the expression in (6) are considered in the context of canonical quantization in $[27]$.

We do not have a description of a basis for the space of vertices. However we can show that the space is non-empty in many cases, by exhibiting a canonical element. This vertex is defined by

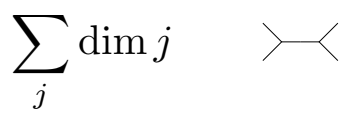


where the middle edge is labelled by $(j, j)$, and is the usual $\mathrm{SU}(2)$ vertex doubled.

This looks unsymmetric because the faces of the tetrahedron have been split into two pairs. Each term in the sum obviously satisfies (2) and (4), and satisfies (3) for the two preferred pairs. The sum of the bivector operators for a pair is equal to the bivector operator for the middle edge in the vertex. This is a simple bivector operator because only simple representations of the form $(j, j)$ appear.

However the pairs can be switched by fusion, with the coefficients determined by the $6 \mathrm{j}$-symbol. The orthogonality relation of the $6 \mathrm{j}$-symbol shows that the vertex is given by the same formula if the top two and bottom two pairs are chosen. For the third splitting, the braidings of self dual and anti self dual pieces cancel, so the same remark applies.

The classical limit. The simple representations of $\mathrm{SU}(2) \times \mathrm{SU}(2)$ occur as the summands of the Hilbert space of functions on $S^{3}$, which has the obvious action of $\mathrm{SO}(4)$. The corresponding classical phase space is therefore the cotangent space $T^{*} S^{3}$. A Lie algebra element $l \in \operatorname{so}(4)$ acts on functions on $S^{3}$ by the formula

$$
l=l^{\mu \nu} x_{\mu} \frac{\partial}{\partial x^{\nu}}
$$

The corresponding momentum in the classical case is $l^{\mu \nu} x_{\mu} p_{\nu}$, for a point $(x, p) \in$ $T^{*} S^{3}$. This is the dual pairing of so(4) with the simple bivector $x \wedge p$ in $\mathbb{R}^{4}$, which explains how simple bivectors are associated to triangles.

This phenomenon is familiar from the standard relativistic angular momentum theory, where the failure of simplicity of the angular momentum is associated with intrinsic spin. In our picture, intrinsic spin occurs for non-simple representations, which would arise from sections of spinor bundles over $S^{3}$.

\section{The NeW ModeL}

It is easy to see how to transform the state sum of [4] to create a quantum version of summation over all assignments of bivector data to the 2-simplexes of a triangulated 4-manifold. We pick labellings of the 2-simplex of the triangulation by irreducible representations of $U_{q}\left(S U_{2}\right)$ just as in [4]. For each such labelling of the 2 -simplexes, we label the tetrahedra in each 4-simplex by elements of a basis for the vector space of vertices defined in the previous section (so that each tetrahedron in the interior of the manifold is labelled twice). In [4], a basis for the space of vertices was determined by specifying a labelling of the tetrahedron by a spin $j$. In the new model, each basis vertex will be a certain linear combination of the $><$

labelled on the middle edge with $(j, j)$.

There are two slightly different possibilities here. The first is to label tetrahedra in each 4-simplex with vertices as just described. The second possibility is to label the tetrahedron in the manifold with

labelled on the middle edge with $(j, j)$ and use this in each of the two 4-simplexes it shares. The extra label $j$ is then summed with the same weights as given by the 
vertex. The second sum is thus the 'diagonal' subset of the first. Thus we actually have two slightly different models, both of which deserve careful study. If vertices other than the canonical vertex exist, there will be still other variant state sums to consider.

In either case, a weight (in $\mathbb{C}$ ) is defined for the labelling, and the state sum model determines a number by summing the weights over all labellings.

For each 4-simplex, a weight is determined by taking the product of all the vertices for its tetrahedra to form a closed relativistic spin network. The weight for the manifold is determined by taking the product of all these weights with correction factors for the simplexes of dimension less than four. For example, on a tetrahedron, this is the inner product between the basis elements in the vector spaces of vertices for the tetrahedron in each of the two 4-simplexes it shares. This ensures that the state sum does not depend on the choice of basis for the space of vertices on tetrahedra in the interior. The lower dimensional corrections would be analogous to the ones in [4], but modified to take into account the fact that we are quantum tracing over two copies of each representation at each step.

In [4] we combined the 15 spins on each 4 -simplex into a diagram called a $15 \mathrm{j}$ q symbol which depended on the orientation of the 4-simplex. Since reversing orientation switches self-dual with anti-self-dual bivectors, in the new state sum the weights are linear combinations of the product of two $15 \mathrm{j}$-q symbols with identical representations but opposite orientation for each 4-simplex of the triangulated 4manifold. Thus the model is a 'constrained' doubling of the Crane-Yetter model.

\section{Conclusions}

The one issue which still needs to be addressed in order for the state sum we are proposing to be a candidate for a quantum theory of gravity is how probability amplitudes computed with it behave as we refine the triangulation. This is analogous to the ultraviolet problems long familiar to quantum field theory and quantum gravity. It is important to note however that the nature of the problem has changed, since we no longer have a continuum metric. Instead of short distance behaviour, we are concerned with fluctuations within fluctuations, with each fluctuation having a characteristic Planck length 'size'. This at any rate is the most natural interpretation of the labellings in our state sum.

There are several possible avenues for constructing a finite theory from our state sum. The most elegant solution would be if there is topological invariance. It will take a good deal of careful calculation of relativistic spin networks to determine if this is the case.

Another possibility is to consider the possibility that below a certain subdivision the universe is in a self-dual, ie topological state. However this would require relaxing the constraints to allow self-dual states in the model. We could think of this hypothesis as related to the idea that the universe began in a topological state.[28]

Another possibility is to try to imitate our construction using either the category of representations of a larger quantum group or possibly a quantum supergroup. It is plausible that the sort of cancellations familiar from supergravity and supersym- 
metry will reappear in our context, and give us a finite theory. The advantage of this line of development over superstring theory is that if the cancellations do give a finite theory, it will be an exact theory rather than a perturbative expansion to something more mysterious.

It is interesting that the sort of tensor categories which go into the state sum we are proposing are so similar to the ones invented in constructing string theories [29]. Terms in our state sum can be interpreted as diagrams in string perturbation theory, by connecting together the diagrams we are associating to the 4-simplexes, and interpreting elements in the representations as string states.

At this point, perhaps the strongest thing which we can say of our model is that it transforms a number of important and familiar questions into statements about finite calculations which are well defined and can be investigated. We believe that this is a good sign in itself.

\section{REFERENCES}

1. L. Crane, Clock and category, is Quantum Gravity Algebraic?, J. Math. Phys 36 (1995), 6180-6193.

2. L. Crane, Topological Field Theory as the Key to Quantum Gravity, Knots and Quantum gravity (J.C. Baez, ed.), Clarendon, Oxford, 1994.

3. J. W. Barrett, Quantum Gravity as Topological Quantum Field Theory, J. Math. Phys. 36 (1995), 6161-6179.

4. L. Crane, L. Kauffman and D. Yetter, State Sum invariants of 4-Manifolds, J. Knot Theory Ram. 6 (1997), 177-234.

5. H. Ooguri, Topological Lattice Models in Four Dimensions Hirosi Ooguri, Mod. Phys. Lett. A7 (1992), 2799-2810.

6. J.C. Baez, Four-Dimensional BF Theory as a Topological Quantum Field Theory, q-alg/ 9507006, Lett. Math. Phys. 38 (1996), 129-143.

7. J. Roberts, Skein theory and Turaev-Viro invariants, Topology 34 (1995), 771-788.

8. J.F. Plebański, On the separation of Einsteinian substructures, J. Math. Phys. 18 (1977), 2511-2520.

9. R. Capovilla, J. Dell, T. Jacobson, L. Mason, Self-dual 2-forms and gravity, Class. Quantum Grav. 8 (1991), 41-58.

10. G.T. Horowitz, Exactly soluble diffeomorphism invariant theories, Comm. Math. Phys. 125 (1989), 417-437.

11. L. Smolin, Linking Topological Quantum Field Theory and Nonperturbative Quantum Gravity, gr-qc/9505028, J. Math. Phys. 36 (1995), 6417-6455.

12. G. Ponzano and T. Regge, Semiclassical limit of Racah coefficients, Spectroscopic and group theoretical methods in Physics (F. Bloch, ed.), North-Holland, 1968, pp. 1-58.

13. E. Witten, 2+1 Gravity as an Exactly Soluble System, Nucl. Phys. B311 (1988), 46-78.

14. C. Rovelli and L. Smolin, Discreteness of Area and Volume in Quantum Gravity, Nucl. Phys. B442 (1995), 593-622.

15. C. Rovelli, The basis of the Ponzano-Regge-Turaev-Viro-Ooguri model is the loop representation basis, Phys.Rev. D48 (1993), 2702-2707.

16. M.P. Reisenberger, C. Rovelli, Sum over Surfaces form of Loop Quantum Gravity, Phys.Rev. D56 (1997), 3490-3508.

17. H. Waelbroeck and J.A. Zapata, A Hamiltonian lattice formulation of topological gravity, Class. Quantum Grav. 11 (1994), 989-998.

18. J.A. Zapata, Topological lattice gravity using self-dual variables, Class. Quantum Grav. 13 (1996), 2617-2634.

19. M.P. Reisenberger, A left-handed simplicial action for Euclidean general relativity, Class. Quantum Grav. 14 (1997), 1753-1770. 
20. J.W. Barrett, A convergence result for linearised Regge calculus, Class. Quant. Grav. 5 (1988), 1187-1192.

21. J.W. Barrett and R.M. Williams, The convergence of lattice solutions of linearised Regge calculus, Class. Quant. Grav. 5 (1988), 1543-1556.

22. J.C. Baez, Spin Foam Models, gr-qc/9709052.

23. A. Barbieri, Quantum tetrahedra and simplicial spin networks, gr-qc/9707010.

24. J.W. Barrett, First order Regge calculus, Class. Quant. Grav. 11 (1994), 2723-2730.

25. J.W. Barrett, M. Rocek, R.M. Williams, A note on area variables in Regge calculus, grqc/9710056.

26. L.H. Kauffman, Spin networks and knot polynomials, Int. J. Modern Physics A 5 (1990), 93-115.

27. J. Lewandowski, Volume and quantizations, Class. Quantum Grav. 14 (1997), 71-76.

28. E. Witten, Topological quantum field theory, Comm. Math. Phys. 117 (1988), 353-386.

29. G. Moore and N. Seiberg, Classical and Quantum Conformal Field Theory, Comm. Math. Phys. 123 (1989), 177-254.

Department of Mathematics, University of Nottingham, University Park, NotTINGHAM, NG7 2RD, UK

E-mail address: jwb@maths.nott.ac.uk

Mathematics Department, Kansas State University, Manhattan KS, 66502, USA

E-mail address: crane@math.ksu.edu 